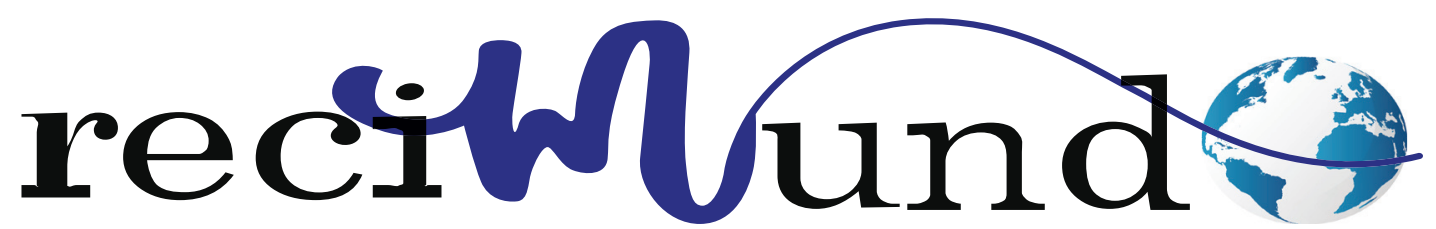

Revista Científica Mundo de la Investigación y el Conocimiento

DOI: 10.26820/recimundo/4.(4).octubre.2020.362-370

URL: http://recimundo.com/index.php/es/article/view/913

EDITORIAL: Saberes del Conocimiento

REVISTA: RECIMUNDO

ISSN: 2588-073X

TIPO DE INVESTIGACIÓN: Artículo de Revisión

CóDIGO UNESCO: Ciencias Médicas

PAGINAS: $362-370$

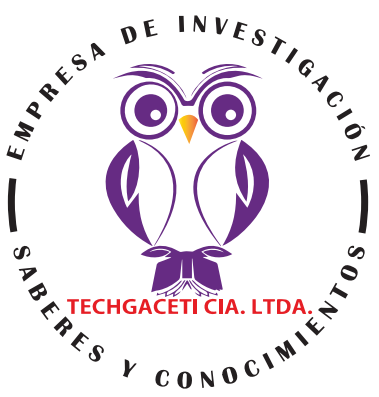

\title{
Síndrome Adherencial: Complicaciones
}

Adherence Syndrome: Complications

Síndrome de adesão: complicações

Adriana Eloísa Grijalva Cifuentes'; Kory Anahi Reinoso Trujillo2;

Rosario Maristhanny García Ferrín ${ }^{3}$ Carlos Roberto Ayora Nolivos ${ }^{4}$

RECIBIDO: 10/07/2020 ACEPTADO: 26/08/2020 PUBLICADO: 15/10/2020

1. Médica General de la Universidad Central del Ecuador; Investigador Independiente; Quito, Ecuador; connie_adri_90@ hotmail.com; (D) https://orcid.org/0000-0002-4459-3851

2. Médico General de la Universidad Central del Ecuador; Médico Residente de Cirugía General del Hospital de Especialidades FFAA N¹; Quito, Ecuador; kory318anahi@gmail.com; (D) https://orcid.org/0000-0002-7191-899X

3. Médico General de la Universidad Central del Ecuador; Médico Residente de Emergencia del Hospital de Especialidades FFAA N¹; Quito, Ecuador; rm.gf.92@outlook.com; iD https://orcid.org/0000-0003-0770-7159

4. Médico General de la Universidad Central del Ecuador; Médico Residente de Cirugía General del Hospital de Especialidades FFAA N¹; Quito, Ecuador; robertoayora@outlook.com; (D) https://orcid.org/0000-0002-2984-259X

CORRESPONDENCIA

Adriana Eloísa Grijalva Cifuentes

connie_adri_90@hotmail.com

Quito, Ecuador

(c) RECIMUNDO; Editorial Saberes del Conocimiento, 2020 


\section{RESUMEN}

El Síndrome Adherencial se refiere al cuadro clínico que se manifiesta cuando las adherencias, que actúan como puentes vascularizado se inervados entretejidos conectivos de órganos, causan complicaciones secundarias importantes. Las adherencias son causantes de muchos casos de infertilidad femenina, obstrucción intestinal y de re-intervención quirúrgica. Considerando que su morbimortalidad ha resultado ser más común de lo que se suele pensar, es fundamental profundizar respecto a su tratamiento, así como su prevención, sobre todo, en aquellos casos que pueden devenir en Síndrome Adherencial, tras la intervención quirúrgica a cavidad pélvico-abdominal abierta. En tal sentido, el presente estudio pretende profundizar en sus causas, tratamiento y sus complicaciones. Las adherencias normalmente no manifiestan ningún signo clínico, de tal forma que hay un alto riesgo de pasar inadvertida. Sin embargo, es importante conocer que las complicaciones causadas por la misma, se manifiestan creando un síndrome típico según sea el caso.

Palabras clave: Síndrome Adherencial, Peritoneo, Dolor abdominal, Obstrucción intestinal.

\section{ABSTRACT}

The Adherential Syndrome refers to the clinical picture that manifests itself when adhesions, which act as vascularized bridges and innervated connective tissues of organs, cause important secondary complications. Adhesions are the cause of many cases of female infertility, intestinal obstruction, and reoperation. Considering that its morbidity and mortality has turned out to be more common than is usually thought, it is essential to deepen regarding its treatment, as well as its prevention, especially in those cases that can develop into adhesion syndrome, after surgical intervention to the pelvic cavityopen abdominal. In this sense, the present study aims to delve into its causes, treatment and complications. Adhesions usually do not show any clinical signs, so there is a high risk of going unnoticed. However, it is important to know that the complications caused by it manifest themselves by creating a typical syndrome as the case may be.

Keywords: Adhesion syndrome, Peritoneum, Abdominal pain, Intestinal obstruction

\section{RESUMO}

A Síndrome Aderencial refere-se ao quadro clínico que se manifesta quando aderências, que atuam como pontes vascularizadas e tecidos conjuntivos inervados de órgãos, causam complicações secundárias importantes. As aderências são a causa de muitos casos de infertilidade feminina, obstrução intestinal e reoperação. Considerando que sua morbimortalidade tem se mostrado mais comum do que se costuma pensar, é imprescindível aprofundar quanto ao seu tratamento, bem como sua prevenção, principalmente nos casos que podem evoluir para síndrome de adesão, após intervenção cirúrgica na região pélvica. cavidade abdominal aberta. Nesse sentido, o presente estudo visa aprofundar suas causas, tratamento e complicações. Geralmente, as aderências não apresentam sinais clínicos, pelo que existe um grande risco de passar despercebida. No entanto, é importante saber que as complicações por ela causadas se manifestam criando uma síndrome típica, conforme o caso.

Palavras-chave: Adhesion syndrome, Peritoneum, Abdominal pain, Intestinal obstruction 


\section{Introducción}

Se habla de Síndrome Adherencial (SA) cuando las adherencias producen dolor y otras series de sintomatologías (dependiendo del lugar donde se han formado), causadas por complicaciones secundarias, caracterizadas generalmente por la obstrucción o disminución de la funcionalidad del órgano afectado por la misma. De hecho, las adherencias son causantes principalmente de muchos casos de infertilidad femenina, obstrucción intestinal y de re-intervención quirúrgica, debido a que la fibrina gelatinosa que actúa como pegamento tratando de reparar un tejido dañado, crean bandas o puentes que comunican tejidos que no deberían estar comunicados (Galindo, 2009)

En consecuencia, su morbi-mortalidad ha resultado ser más común de lo que se suele pensar, fundamentalmente aquellas que devienen de intervenciones quirúrgicas, siendo la de mayor incidencia las producidas a partir de las cirugías pélvicas abiertas en un $90 \%$ de los casos, aproximadamente, y en un promedio de $75 \%$ de los casos por cirugía abierta de cavidad abdominal (Gaviria P, Herrera I, Hernández S, Gutiérrez P, \& Gaviria P, 2017)

Debido a que las adherencias pueden ser creadas por el organismo en cualquier parte del mismo, el estudio de las éstas puede especializarse de diversa manera. Sin embargo, para efectos del presente artículo, se presenta de manera general la patogénesis de dicha patología, y se enfoca principal atención en las adherencias abdominales y pélvicas, por ser estas las manifestaciones mayor incidencia, así como por su propensión a generar complicaciones.

En tal sentido, es importante comprender esta patología desde un enfoque preventivo, y no sólo desde el tratamiento, sobre todo, aquellas que pueden devenir tras la intervención quirúrgica, como es el caso de las adherencias peri-abdominales, que pudieran haber iniciado a partir de la reacción cicatricial exagerada ante cuerpos extraños, o ante una incisión, sutura, o electrocoagulación y cuerpos extraños, produciendo una liberación de sustancias vasoactivas y citocinas, generada por monocitos que actúan en el sitio del evento, luego llevando a la vasodilatación y aumento de la permeabilidad vascular, creando un exudado rico en fibrina. Se conoce que se produce dentro de las primeras 12 horas, en las que aparece una densa capa de fibrina infiltrada con leucocitos polimorfos nucleares; entre las 24 y las 36 horas de iniciado el proceso aparecen macrófagos sobre la capa de fibrina. En el día 8, estas células mesoteliales se encuentran firmemente unidas a la membrana basal del segmento lesionado" (Valdivia Gomez, Tena Betancourt, \& de Alva Coria, 2019)

El presente artículo presenta al SA comprendiéndolo desde su etiopatogenia, para explicar brevemente los diferentes tipos y clasificación, así como los aspectos clínicos más importantes del SA abdominal, por ser más típico y causal de la mayoría de las complicaciones, así como los factores asociados.

\section{Metodología}

Para el desarrollo del presente artículo se realizó una exhaustiva documentación y recolección de datos sobre el Síndrome Adherencial (SA), partiendo de los conceptos específicos hasta la fundamentación general. Para ello, se abordaron literaturas científicas valorando la calidad y veracidad de la información recopilada, desglosando e indagando terminologías médicas.

Sumado a ello, dentro de éste enfoque documental descriptivo adoptado para la elaboración del documento, se apeló por la experiencia de profesionales médicos que corroboraron, descartaron, y agregaron información, para finalmente, obtener la orga- 
nización de los datos. Dicha organización de la información levantada fue reestudiada a fin de aclarar terminologías aplicadas dentro del artículo, refiriéndose al Síndrome Adherencial (SA) como al conjunto de sintomatología desplegada a partir de la complicación Adherencial, más específicamente, en las cavidades abdominales y/o pélvicas, ya que las adherencias puede ocurrir en cualquier parte del cuerpo, incluyendo en la musculatura ocular luego de una cirugía.

\section{Resultados}

\section{Adherencia}

El termino adherenciasproviene del latín tardío «adhaerentia»[ad «junto a» + haerē(re) "adherirse» + -ntiam «acción de»] que alude al hecho de que son puentes vascularizados e inervados de tejido conectivo entre órganos. Son fibrinas, que derivan del término brida tomado del francés «bride», y del germanismo bridel, («brit’l o «brîtil»), que se acumulan sobre el tejido dañado comportándose como un pegamento para aislar el daño .

Las adherencias normalmente no manifiestan ningún signo clínico y muchas veces pasa inadvertida. Sin embargo, otras causan complicaciones creando un síndrome típico según sea el caso. Por ejemplo, las adherencias hepáticas causan dolor cuando el paciente inhala y exhala profundamente, mientras que las inter o peri intestinales tienden a causar más dolor con ejercicio o estirar.

Algunos estudios epidemiológicos revelan que el grupo etario que más frecuentemente presenta esta patogenia son pacientes mayores de 45 años (63\%) y de sexo femenino (63\%), debido a las adherencias pélvicas, donde las bridas obstruyen asas intestinales. En efecto señala (Palacios Silva, León Rodas, Palacios Silva, \& Gúzman Lozada, 2018) que el $37,9 \%$ generalmente son casos de cirugía previas gastrointestinales. Además, $45 \%$ de los casos de SA que generan mayor complicaciones son los casos de obstrucción (33\%), seguido de isquemia $(12,1 \%)$, luego perforaciones (8\%) y en último lugar abscesos y fistulas (5,8\%-1,1\%). La edad promedio de pacientes que consultan por bridas sintomáticas está entre 25 y 50 años. La reaparición de adherencias intra-peritoneales sintomáticas, posterior a un procedimiento de adhesiolisis se estima en 11 a 30\%. La mortalidad atribuida a las adherencias se correlaciona principalmente con la obstrucción intestinal y con el procedimiento de adhesiolisis, que corresponde entre 6 y $11 \%$.

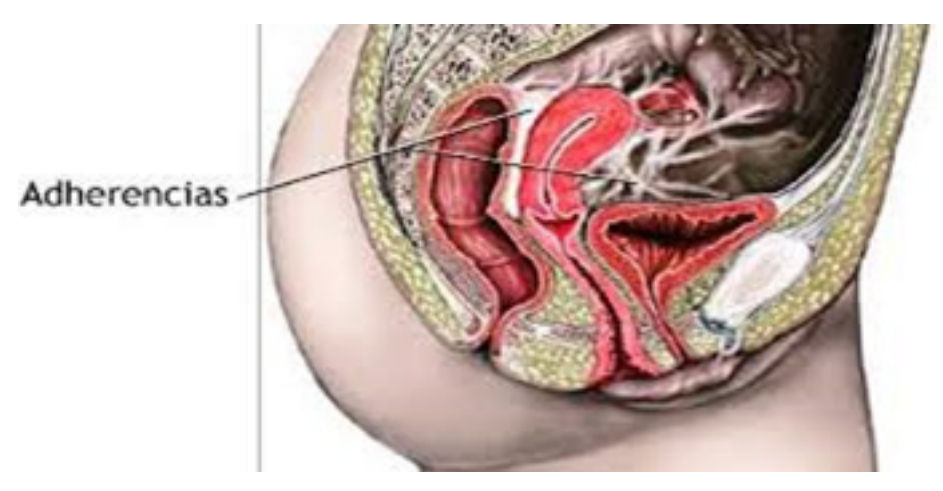

Figura 1. Adherencias

Fuente: (Moreno Barrera \& Ramírez Robinson, 2020)

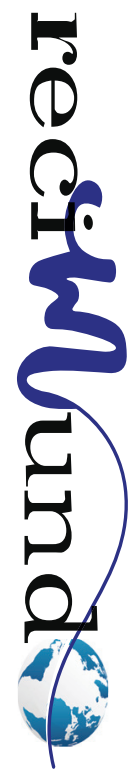




\section{Etiopatogenia}

Las adherencias son el resultado final de un desequilibrio entre el proceso de fibrinólisis y fibrogénesis, dicho descontrol conjuntamente con hipoxia tisular secundaria a daño mesotelial, que genera mayor respuesta inflamatoria del tejido lesionado y un aumento de la población de los fibroblastos de adherencia y de la inhibición de la degradación de la matriz extracelular (Valdivia Gomez, Tena Betancourt, \& de Alva Coria, 2019).

Una vez iniciada la lesión tisular, que puede originarse ya sea por una incisión, procedimiento durante la cirugía, por daño procedentes de radiación, infecciones abdominales o pélvicas, ente otros, se liberan citocinas, producidas por los monocitos que actúan en el sitio. El fibrinógeno vuelve insoluble interactuando con proteínas grandes incluyendo fibronectinas, produciendo, de esta manera, la matriz gelatinosa de fibrina.

Esta fibrina actúa como pegamento para aislar el daño, así construye adherencia conectando tejidos adyacentes. En la natural fisiología, esta acumulación de fibrina debería ser controlada por las enzimas que limitan o disuelven su índice de producción. No obstante, en el caso de tejido cicatrizante, este proceso es incompleto, resultando en adherencia, formada principalmente por las células de la reparación, tales como: fibroblastos y macrófagos, las cuales producen colágeno para crear una estructura cada vez más fuerte en un lapso de tiempo, y puede llegar a ser permanente,"o destruida en 12 horas relacionado este efecto a la deficiente actividad fibrinolítica" (Elizondo Hinojosa, López Gutiérrez, Poblano Morales, Yañes López, \& Pérez García, 2004)

Como ya se ha descrito, la causa más frecuente de adherencias abdominales es por cirugía. Los eventos durante la cirugía que conducen a la formación de adherencia involucran procedimientos incisión, la deshidratación de órganos y de tejidos abdo- minales, cuerpos extraños como guantes con los tejidos internos, entre otros. Efectivamente, órganos y estructuras dentro del abdomen, como el epiplón, son considerados como los más susceptibles a la formación Adherencial.

La defensa natural más importante contra la formación de adherencias es el activador del plasminógeno (tAP); la cual descompone la matriz gelatinosa de fibrina deteniéndose así el efecto de formación de adherencias. En el primer día del postoperatorio, la actividad de tAP en el líquido de lavado peritoneal no disminuye, lo que es importante, ya que el gran aumento de fibrina que es observado, indica que es insuficiente el rango que minimiza la actividad fibrinolítica, siendo esta la principal causa de acumulo de fibrina posterior al trauma peritoneal.

De acuerdo con (Elizondo Hinojosa, López Gutiérrez, Poblano Morales, Yañes López, \& Pérez García, 2004), existe una reducción postquirúrgica de la actividad fibrinolítica, por tres caminos:

- En el sitio de la lesión, quirúrgico o inflamatorio se incrementan los niveles de inhibidores de la activación del plasminógeno (PAI 1 y PAI 2).

- Se disminuyen los niveles de activador del plasminógeno (tAP).

- En el postquirúrgico se produce una marcada reducción de la oxigenación tisular.

Y añade, los dos eventos claves en la formación de adherencias:

1. La coaptación de las dos superficies mesoteliales dañadas.

2. El desbalance entre los depósitos de fibrina y su disolución.

\section{Clasificación de las adherencias}

Se pueden clasificar de acuerdo a las siguientes categorías: 
- Clasificación de las adherencias según su origen

- Clasificación de las adherencias por su localización anatómica.

- Clasificación de las adherencias según su presentación

\section{Clasificación de las adherencias según su origen}

a) Adherencias Congénitas: se producen por anormalidades en el desarrollo de la cavidad peritoneal del embrión.

b) Adherencias Adquiridas: pueden ser producto de procesos inflamatorios o aparecer a raíz de un posterior a intervenciones quirúrgicas abdomino-pélvicas. También por el desarrollo de patologías agudas intra-abdominales tales como apendicitis, colecistitis, Diverticulitis y enfermedad pélvica, así como el uso de dispositivos intrauterinos, que representan la causa principal de la creación de adherencias adquiridas por procesos inflamatorios.

Las adherencias postquirúrgicas son consecuencia de la inhibición de la fibrinólisis, depósitos de colágeno, neo-vascularización y disminución de la actividad del factor activador de plasminógeno (Gaviria P, He- rrera I, Hernández S, Gutiérrez P, \& Gaviria $P, 2017)$

Clasificación por localización anatómica:
a) Viscero Parietales.
b) Viscero viscerales.
c) Viscero Epiploicas.
d) Epiploico Parietales.

Sin embargo, en términos de patogénesis, se pueden mencionar, dos tipos de adherencias: las que se forman después de un trauma simple y las que se desarrollan después de un evento isquémico o inflamatorio, para esta última, el valor clínico de dicha clasificación tiene como objetivo su prevención.

La mayoría de las adherencias que se forman después de una cirugía no están en relación con el sitio de la cirugía, "pero si están involucradas las asas del intestino delgado. Estas adherencias entre asas son la principal causa de obstrucción intestinal y probablemente, son producidas por trauma local simple que disminuye parcialmente los niveles de AP tisular en el momento de la cirugía" (Moreno Barrera \& Ramírez Robinson, 2020)

Tabla 1. Clasificación de adherencias peritoneales según su presentación

\begin{tabular}{|l|l|}
\hline 0 & Ausencia de Adherencia. \\
\hline 1 & $\begin{array}{l}\text { Solo una banda de adherencias entre vísceras o hacia la } \\
\text { pared abdominal. }\end{array}$ \\
\hline 2 & Dos bandas entre vísceras o hacia la pared abdominal. \\
\hline 3 & $\begin{array}{l}\text { Más de dos bandas entre vísceras o hacia la pared abdominal, } \\
\text { o formando una masa sin adhesión a la pared. }\end{array}$ \\
\hline 4 & $\begin{array}{l}\text { Vísceras adheridas directamente a la pared abdominal } \\
\text { independientes del número de bandas. }\end{array}$ \\
\hline
\end{tabular}

Fuente: (Gaviria P, Herrera I, Hernández S, Gutiérrez P, \& Gaviria P, 2017) 


\section{Síndrome Adherencial y Complicaciones}

Generalmente se presente de forma asintomática, pero cuando se manifiesta, viene acompañada, con obstrucción en el intestino y dolencia. Sin embargo, tal como plantea (Galindo, 2009) los síntomas no necesariamente guardan relación con el número de adherencias "sino con la disposición que tienen y trastornos mecánicos que desencadenan".

De tal forma, que dentro de los síntomas que requieren la atención médica inmediata, se incluyen la incapacidad para expulsar gases intestinales o para defecar, una distensión progresiva del abdomen, acompañado por los sonidos ruidosos del intestino, un dolor abdominal severo asociado a obstrucción.

Obstrucción Intestinal: es la complicación más frecuente de esta patología, la cual se genera por alteraciones en el tránsito intestinal, que se manifiesta en uno o varios sectores.

Entre los mecanismos se encuentran:

- Angulación

- Constricción

- Torsión del intestino

La misma se manifiesta de forma aguda o de manera intermitente; muestra mejoría con disminución de ingesta. El dolor acompaña a la distensión abdominal, ruidos hidro- aéreos que indican el pasaje del contenido intestinal en zonas estrechas mejorando la sintomatología.

Dolencias: es la manifestación más representativa que se expresan principalmente en las adherencias ginecológicas, mediante el dolor pélvico crónico, que puede ir acompañado de dispare unía e infertilidad. (Galindo, 2009)

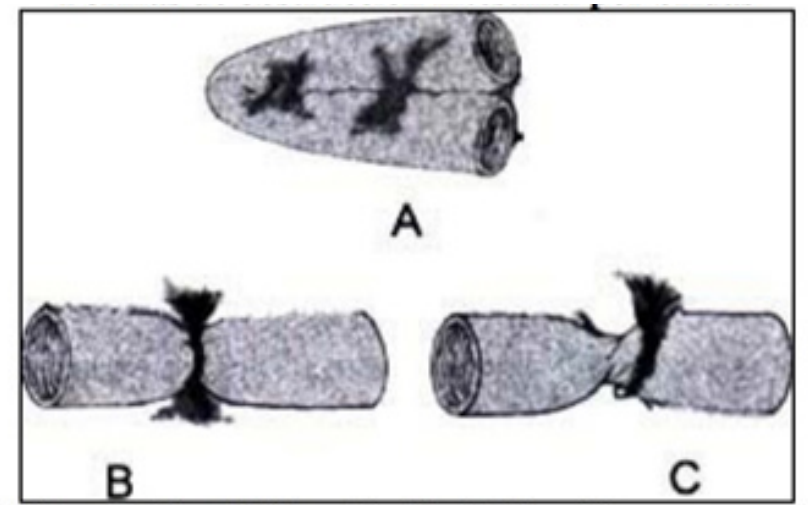

(A) Angulación. (B) Constricción. (C) Torsión.

Figura 2. AFormas de obstrucción intestinal por bridas

Fuente: Rev. Europan Journal Surgical

\section{Tratamiento preventivo}

El tratamiento preventivo se mencionan los siguientes cuidados, que tal como señala (Galindo, 2009) deben considerarse por el cirujano y del paciente:

- Manipulación correcta de los tejidos

- Hemostasia correcta, mediante el uso del tipo de material adecuado para las suturas en concordancia con cada caso particular, que sea reabsorbibles.

- Se debe mantener el peritoneo humedecido si ha estado expuesto por tiempo prolongado al exterior.

- Evitar dejar cuerpos extraños, tales como talco, gasas y otros.

- Peritonizar las zonas que han sido desprovistas de la serosa.

- Lavado de la cavidad peritoneal. Se debe utilizar solución fisiológica 0,9\% a $37^{\circ} \mathrm{C}$. Las temperaturas más elevadas lesionan e incrementan la posibilidad de adherencias.

\section{Tratamiento de la Obstrucción por Adhe- rencias}

\section{Tratamiento Médico}

El tratamiento médico debe intentarse para dos razones, "el tratamiento quirúrgico, 
aunque necesario en muchos casos, nunca nos pone al abrigo de la formación de nuevas adherencias. El tratamiento médico es efectivo entre $30 \%$ a $50 \%$ de los casos" (Galindo, 2009); esto principalmente, porque descomprime la distensión, con la intención de que las asas intestinales se ajusten y recuperar el transito, mientras se utiliza la vía parenteral para mantener el equilibrio del medio interno.

Sólo los pacientes con una obstrucción parcial son sometidos a tratamiento médico, y no deberán presentar signos de compromiso vital (peritonitis, estrangulación intestinal). Se comenzará evitando la ingesta por la boca y colocando una aspiración naso-gástrica y administrando soluciones parenterales para mantener el equilibrio hidro-salino.

"La recuperación del peristaltismo posibilitara la ingesta de líquidos, siguiendo con alimentos blandos de fácil digestión y pobres en residuos. Los pacientes que reinciden con el cuadro de obstrucción son candidatos para la cirugía" (Galindo, 2009)

\section{Tratamiento Quirúrgico}

Consiste en la liberación o seccionar adherencias para recobrar el tránsito intestinal y para evitar que el intestino se vuelva a adherir de una forma desordenada, de tal manera que origine nuevas obstrucciones. En tal sentido, se han ideado cirugías de fijación del intestino que se conocen como enteropexia:

"Si existen zonas comprometidas en su vitalidad deberán ser resecadas. Las obstrucciones por adherencias se producen en intestino delgado. En colon son mucho menos frecuentes" (Galindo, 2009)

\section{Conclusiones}

Las AAP son una causa de dolor abdominal crónico, obstrucción intestinal e inferti- lidad en las mujeres. Hasta ahora, el tratamiento consiste en la liberación quirúrgica de las adherencias, sin embargo, esto a su vez va acompañado de aumento del riesgo de recurrencia.

La prevención de la formación de las AAP está encaminada a disminuir el daño mesotelial con el manejo gentil de los tejidos y evitar el uso exagerado del electrocauterio y de material de sutura. El Síndrome Adherencial es una patología prevenible, sin embargo su incidencia, costo, y mortalidad son altas, incluso cuando se cuentan con diferentes formas para disminuir su presentación. A pesar de todo esto, está claro que el uso de algunos de los métodos presentados, disminuye de una u otra forma su aparición y gravedad, aún y cuando los diferentes estudios no lleguen a un acuerdo en el porcentaje de reducción.

Uno de los retos que tiene actualmente el médico especialista es profundizar en el conocimiento epidemiológico de esta patología, y en su comportamiento a través del tiempo, porque aquí pueden radicar las claves que nos orienten hacia la investigación de medidas de tipo práctico para combatirla, que actualmente se centra casi exclusivamente a lo que se refiere a su profilaxis. De igual forma, es menester el desarrollo de método de diseño de estudios que nos aporte evidencias claras sobre el resultado de tales medidas.

\section{Bibliografía}

Calabuig G, M. (2018). ¿Bridas, Adherencias o Sinequias? Cirugía Española, 96(9), 598-601.

Duboscq, C. (Agosto de 2017). El sistema plasminógeno plasmina. HEMATOLOGÍA, 21, 48-55.

Elizondo Hinojosa, J. L., López Gutiérrez, J., Poblano Morales, M., Yañes López, J., \& Pérez García, R. (2004). Ahherencias Peritoneales Postquirurgicas: Fisiologia y Prevencion. Rev. Hosp Jua Mex, 71(1), 36-42.

Galindo, F. (2009). Adherencias peritoneales. Cirugía Digestiva(II), 1-8. 
Gaviria P, J., Herrera I, A., Hernández S, R., Gutiérrez P, C., \& Gaviria P, J. (2017). Síndrome Adherencial: Prevención Holística. Revista Semilleros Med, 11(1), 33-37.

Liakakos, T., Thomakos, N., Dervenis, C., Young, R. L., \& Fine, P. M. (2001). Peritoneal adhesions: etiology, pathophysiology, and clinical significance. Recent advances in prevention and managment. Dig Surg, 18(4), 260-273.

Moreno Barrera, E., \& Ramírez Robinson, R. (2020). Síndrome adherencial. Presentación de un caso, hospital "Luis Vernaza" de Guayaquil. MEDICINA, 52-56.
ORGANIZACIÓN MUNDIAL DE LA SALUD (OMS). (2016). Estrategia Mundial del Sector de la Salud Contra las Hepatitis Víricas 2016-2021. Hacia el fín de las hepatitis Víricas. WHO Document Production Services, Genova, Switzerland.

Palacios Silva, K. V., León Rodas, R. M., Palacios Silva, A. E., \& Gúzman Lozada, J. A. (2018). Comportamiento de las Complicaciones en el Sindrome Abherencial. Archivos Venezolanos de Farmacologia y Terapeutica, 212-217.

Valdivia Gomez, G. G., Tena Betancourt, E., \& de Alva Coria, P. M. (2019). Adherencias Adbomianales Postoperatorios: Patogenesis y Técnicas Actuales de Prevención. Cirugia Cirujanos, 698-703.

\section{CITAR ESTE ARTICULO:}

Grijalva Cifuentes, A. E., Reinoso Trujillo, K. A., García Ferrín, R. M., \& Ayora Nolivos, C. R. (2020). Síndrome Adherencial: Complicaciones. RECIMUNDO, 4(4), 362-370. https://doi.org/10.26820/recimundo/4.(4).octubre.2020.362-370

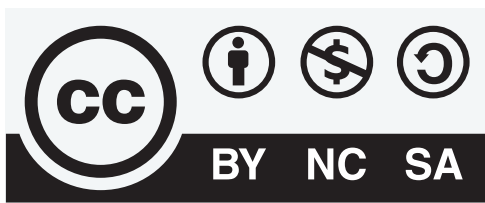

CREATIVE COMMONS RECONOCIMIENTO-NOCOMERCIAL-COMPARTIRIGUAL 4.0. 